\title{
Participación y racionalización en el área de recursos humanos: reforma de la gestión y el empleo público en Suecia
}

Lennart Gustafsson *

\section{Reforma de la gestión pública bajo condiciones económicas cambiantes}

En Suecia, el sector público está experimentando el período más espectacular de cambios que jamas haya conocido, tanto desde el punto de vista internacional como desde el contexto histórico del país, que se encuentra ahora en el umbral del proceso de revisión más amplio del sector público desde la década de los años sesenta. Los progresos de entonces se produjeron, no obstante, en sentido opuesto. El sector público sufrió en esos años una expansión que, hasta hoy, no admite paralelismos. Los suecos se enfrentan ahora a un doloroso proceso de reajuste, durante el cual deberá reducirse el nivel del gasto público. La idea de la necesidad de acometer esta reforma va ganando adeptos de forma gradual. Además, la pertenencia a la Unión Europea supone para el sector público una serie de nuevos retos que afectan a las políticas económicas (a causa de los criterios de convergencia) y demandan nuevas formas de relación entre las Administraciones nacionales y las instancias internacionales.

\section{El desempleo alcanza niveles récord}

Suecia se enfrenta ahora a niveles de desempleo muchísimo más altos de los que se podrían haber previsto hace sólo cinco años. Para los suecos, un nivel de desempleo de un tres por ciento se considera tradicionalmente como algo excesivo. Hoy, aproximadamente el 12 por ciento de la población con edad para trabajar se encuentra fuera del mercado de trabajo ordinario. De otra parte, las expectativas indican que, durante los próximos tres años, esta cifra sólo disminuirá levemente a pesar de que las empresas exportadoras están cumpliendo bien sus objetivos. La mejora en la productividad es parte de la explicación.

\section{El enorme déficit presupuestario debe eliminarse}

$\mathrm{Si}$ bien es el desempleo lo que preocupa más a la gente, existen otras manifestaciones de la crisis económica que son tan serias como la primera. El déficit del presupuesto del Estado asciende ahora a poco más menos del 12 por ciento y la deuda nacional supone aproximadamente el 90 por ciento del producto nacional bruto. El gobierno no controla su propia mayoría en el Riksdag (Parlamento). El recién elegido gobierno socialdemócrata presentó un programa económico a la Cámara, pero todavía existen dudas de que el sistema político pueda ser lo suficientemente fuerte como para hacer que las finanzas públicas recuperen su salud.

\section{Divisa débil y tipos de interés altos}

Por encima de todo lo anterior, los tipos de interés internacionales son relativamente altos, lo que, entre otras cosas, está relacionado con la grave crisis que viene padeciendo el mercado inmobiliario desde hace algunos años; pero principalmente se deben a las dificultades que tiene el gobierno para confeccionar políticas económicas creíbles en el ámbito nacional e internacional. La corona sueca fluctúa y ha perdido un 25 por ciento respecto a las divisas más importantes en menos de tres años. Todavía parece haber mucho escepticismo internacional respecto al futuro de la economía sueca.

\section{La globalización de la economía a largo plazo}

Esto significa que la Administración sueca no puede afrontar los cambios de forma aislada, sin tener en cuenta el entorno de las finanzas públicas de los demás países. Las condiciones económicas cambiantes han modificado el contexto en que se mueve el sector público y han establecido nuevas fronteras entre las esferas de lo privado y de lo público.

Esta evolución tiene en parte su origen en el aumento de la competitividad entre naciones que siguió a la desregulación del sistema económico en los años ochenta y en su coincidencia con la total libertad para la movilidad del capital en el ámbito internacional.

En una economía universalizada, un país no puede soportar la carga que supone un sector público ineficaz y sobredimensionado. Tal país perdería inevitablemente competitividad respecto al resto. En este sentido, cada vez se entiende más la importancia de poseer un sector público adecuadamente dimensionado y que funcione bien como un arma esencial frente a la competencia internacional. 
La cuestión se centra abora no sólo en el nivel de los servicios y la eficacia que ofrece la Administración, sino también en la forma misma del Estado del bienestar

Las nuevas circunstancias económicas externas han generado un también nuevo debate político sobre el futuro del sector público. A diferencia de la polémica que se suscitó hace diez años en Suecia y en otros países con relación a la modernización del sector público, ahora se discuten cambios estructurales fundamentales.

El programa de modernización del sector público se inició con seriedad tras el retorno al poder de los socialdemócratas en 1982. La renovación de este sector afectó entonces a los métodos de funcionamiento de la Administración. El gobierno no socialista que se instauró en 1991 planteó la necesidad de llevar a cabo la reforma de otra manera y en el mismo partido socialdemócrata, que volvió a ganar las elecciones en 1994, se están suscitando también cuestiones de gran importancia en este sentido. Desde hace ya unos años, el debate está tomando un cariz totalmente nuevo en Suecia porque es el legendario Estado del bienestar lo que se encuentra encima de la mesa.

A principios de la década de los noventa, se empezó a formar un consenso político en torno a los costes excesivos que tal Estado del bienestar suponía si se quería conseguir una economía equilibrada. Las finanzas públicas tenían que andar por el buen camino y esto afectaría el nivel de compromiso del Estado con la población. Se intenta que la proporción del PNB destinada a gasto público disminuya y esto tendrá una repercusión tanto en el volumen de las transferencias como en índice de consumo. Se pretende, de diversas maneras, dejar más funciones al mercado, sin que el Estado intervenga. La cantidad de dinero que se canaliza a través de las instituciones del sector público sufrirá un relativo descenso.

Es, sin embargo, no sólo cuestión de solvencia en los pagos del Estado y de los municipios. Los sistemas del bienestar, con sus seguros de enfermedad, seguros de desempleo y protección familiar se ven cada vez más como algo incompatible con las exigencias que se derivan de la necesidad de mantener la productividad dentro de unos márgenes que permitan el crecimiento y la competitividad internacional. Se considera que estos sistemas no estimulan ni la eficacia ni el crecimiento.

Además, las concepciones sobre gestión y eficacia del sector privado han ganado terreno últimamente en la Administración pública. Fueron otros los ideales sobre los que anteriormente se basaron los criterios de organización y administración.

\section{Redefinición del papel del sector público}

Estas transformaciones están originando en los últimos años una concentración deliberada de las labores del sector público. Bajo el gobierno no socialista (1991-94), el Estado se retiró de aquellas actividades que el mercado podía asumir. No es misión del Estado intervenir en áreas abiertas a la competencia. Como primer paso, las telecomunicaciones, Correos y el sector energético se reorganizaron bajo una estructura empresarial. Todavía son propiedad del gobierno, pero es de esperar que, en un futuro, se produzca al menos una privatización parcial en las mismas.

En ciertos casos, este proceso de concentración se produce de forma drástica. Algunos organismos estatales han sido eliminados, no porque fueran ineficaces o cumplieran mal su cometido, sino porque se ocupaban de actividades que el gobierno no consideraba asunto del Estado.

La agencia pública dedicada a la formación de los funcionarios es un ejemplo. Esta función es responsabilidad de los jefes de cada unidad (agencia o departamento) y éstos se dirigen a las instituciones educativas o a empresas de asesoramiento que operan en el mercado en aquellos casos en que consideran que ellos mismos no pueden facilitar la formación adecuada a su personal.

La competencia como razón primera incluso en aquellas áreas donde el Estado sigue ostentando un control absoluto

Otro cambio en la forma de proceder de los organismos públicos es la presencia de la competitividad y los principios de mercado como motor incluso en aquellas áreas donde el gobierno local o central es el único responsable de proporcionar un determinado servicio a los ciudadanos. En muchas ocasiones, la manera en que se han concedido las ayudas ha llevado a la creación de monopolios (a todos los efectos) en áreas como la atención infantil y la hospitalaria. Este monopolio se ve ahora disminuido porque se están concediendo ayudas en las mismas condiciones a través de competidores del sector privado.

La relaciones contractuales o de compra-venta se están convirtiendo en algo habitual en los ayuntamientos suecos a la hora de satisfacer los compromisos adquiridos con los ciudadanos.

No obstante, tras haber probado este método durante algunos años, la cuestión de la eficacia real forma parte ya del orden del día de la reforma de la gestión pública.

La subcontratación se aplica también en áreas de responsabilidad tradicionalmente estatales, como la de la red viaria, donde el Estado conserva una responsabilidad financiera en la construcción y mantenimiento, pero son empresas privadas las que realizan el trabajo. Esta circunstancia provoca no pocos problemas de control de gestión a la administración de la red nacional de carreteras -además de generar una prestación de servicios de gran número de personal que ya no se necesita.

\section{Reforma de las ordenanzas en un contexto nuevo}

La reforma legislativa es una parte fundamental del programa de reformas del sector público en la mayoría de países de la 
OCDE. Este proceso presenta habitualmente dos vertientes: la reducción del número de ordenanzas que regulan las diversas actividades de los individuos y de las empresas y la simplificación de las que se necesita conservar. La intención última que ha inspirado desde el principio estos procesos ha sido la de hacer la vida más fácil a los ciudadanos. Los programas han formado parte de lo que se ha venido en llamar servicio de iniciativas de gestión. Desde los años setenta, Suecia ha desarrollado pacientemente estos programas y, en el transcurso del tiempo, se han conseguido simplificaciones considerables.

Este proceso continúa, pero los nuevos modelos de transformación han impuesto a su vez objetivos nuevos en la simplificación reglamentaria. El mercado de trabajo, las comunicaciones, los mercados financieros, el mercado inmobiliario y las compañías de electricidad han sido en conjunto objeto de una desregulación. Se ha simplificado la legislación relativa a impuestos y a educación. La diferencia, ahora, estriba en que el objetivo es la creación de un sistema normativo que mejore la capacidad competitiva y el indice de crecimiento.

\section{Nuevas prioridades para el gasto público}

El cambio de dirección que se observa en la reforma legislativa afecta también a las prioridades establecidas para el gasto público, el cual permanecerá en el marco de unos límites presupuestarios en constante reducción. Durante los años de expansión de la década de los sesenta y de las dos décadas siguientes, la bonanza económica repercutió en todas las áreas. Se tomaron iniciativas importantes en los sistemas de transferencia y en los servicios municipales. "Bienestar" sería la palabra que podría resumir los incrementos del gasto público sueco que tuvieron lugar en ese período.

Hoy, el objetivo de las iniciativas que se toman es la mejora de la competitividad nacional y de la tasa de crecimiento. En un sentido práctico, esto significa que el dinero se gastará en mejorar la infraestructura física y en nuevas propuestas para la investigación y el desarrollo.

En resumen, puede decirse que el centro de atención de las nuevas políticas destinadas a la renovación del sector público se ha desplazado del desarrollo de la Administración como un aparato más eficaz para dispensar un cierto número de servicios -cuya existencia no era discutida- a la transformación del papel que ha de jugar el sector público en el conjunto de la sociedad. Las reformas administrativas de los años ochenta no han perdido su importancia; su peso relativo, no obstante, ha disminuido porque nunca pudo conseguir los "recortes" que ahora se exigen para el sector público.

Este nuevo contexto ha sido posiblemente el causante de que se desatendieran reformas administrativas importantes en el debate público. El proceso de ajuste que se está llevando a cabo en organismos y departamentos gubernamentales es, no obstan- te, más pronunciado que nunca. Un ejemplo de ello es que se está produciendo una reducción sin precedentes del número de empleados públicos.

\section{La estructura y la gestión de la Administración} estatal

Desplacemos ahora nuestra perspectiva de la discusión de las nuevas condiciones que limitan la acción del gobierno para observar qué repercusiones han tenido tales condiciones cambiantes en la estructura y la gestión de la Administración del Estado. Sorprende apenas ver que una característica recurrente es conseguir que las agencias gubernamentales produzcan más barato y mejor. Los organismos satisfacen esta premisa de varias formas.

\section{Diversas formas de aborrar}

En primer lugar, los gobiernos han asumido durante mucho tiempo que los presupuestos de sus Administraciones tenían intrínsecamente un potencial de "ahorro". La mayor parte de esas Administraciones, por tanto, han contemplado medidas de ahorro en la asignación de fondos que eran fijadas por el gobierno. Sería complejo argumentar que tales previsiones de ahorro se cumplieron siempre de forma científica. No es inusual que el gobierno asuma que las agencias estatales deban conseguir una mejora en su productividad de al menos un dos por ciento anual. Partiendo del análisis de los presupuestos, se asume que la producción puede mantenerse al mismo nivel con un dos por ciento menos de recursos reales de un año al otro.

Este instrumento de control de gestión está considerado por todos como algo demasiado contundente y simplista que conlleva muchos riesgos de asignación incorrecta de recursos. A pesar de todas sus deficiencias, se utiliza todavía, al tiempo que se estudia la posibilidad de introducir métodos más modernos de gestión por resultados.

Las previsiones de aborro contemplan una importante delegación de poderes

Dentro de sus menguados presupuestos, las instituciones y departamentos gozan de una considerable libertad para reorganizar sus métodos de producción en función de los objetivos marcados. Esta delegación de autoridad es claramente una condición para conseguir un aumento de la productividad. Cabe hacer mención aquí que estas instituciones o agencias son el equivalente organizativo a las divisiones internas de los ministerios de otros países. Comparadas con éstas últimas, las primeras han gozado durante mucho tiempo de una gran independencia en Suecia. 
Las reducciones presupuestarias no bastan -se bacen necesarios cambios estructurales

A lo largo de la década de los ochenta, el gobierno creía que el aumento de la eficacia podía alcanzarse mediante una disminución de recursos acompañada de mayor libertad para los gestores para poner en marcha los cambios necesarios. El gobierno estaba para fijar el rumbo del barco en su viaje hacia la modernización y crear el marco financiero pertinente. A partir de ahí, cada gestor continuaba el trabajo.

El problema de esta manera de funcionar era que los gestores, por razones obvias, se veían limitados a cambiar sólo las estructuras que ellos gestionaban. Había, por decirlo de otra manera, un listón para los logros ya establecidos según la división de funciones definida para los distintos organismos gubernamentales. Esta filosofía descentralizadora se complementó consiguientemente con una visión neocentralista. A los recortes presupuestarios de los años ochenta en ciertos organismos se unieron progresivamente controles del sector central, donde comités nombrados por el gobierno inspeccionaban la estructura global de las agencias con responsabilidades en áreas operativas estrechamente relacionadas.

Estas inspecciones daban a menudo como resultado la transferencia de algunas funciones y responsabilidades del sector central hacia cuerpos administrativos locales o regionales. Este fue el caso de la policía, las autoridades aduaneras, las prisiones y la administración de la libertad condicional, instituciones del mercado laboral, mantenimiento de la red viaria y las agencias tributarias, por mencionar sólo las más significativas de ámbito nacional.

Además de estos controles sectoriales, el nuevo gobierno ha anunciado una revisión en profundidad del sector público y su Administración, en que la cuestión primordial será el papel que debe desempeñar el Estado y la forma en que debe diseñarse la estructura organizativa para cumplir los cometidos que se le asignen en función del estudio.

\section{La gestión por resultados es la reforma administrativa más importante}

El proyecto más ambicioso es sin duda la introducción de un nuevo sistema de gestión y confección de presupuestos. De todas las reformas emprendidas por el gobierno, es quizás la implantación de una gestión por resultados en la relaciones del gobierno con sus agentes institucionales la que hasta el momento ha producido los mayores efectos estratégicos a largo plazo en las políticas de la Administración pública.

Todo sistema de gestión por resultados aspira a trasladar el centro de atención de las entradas presupuestarias a los resultados operativos. Este fue el caso también de Suecia en los años setenta cuando intentó poner en práctica un programa presupuestario que desglosaba los objetivos generales en un cierto número de logros parciales en cada nivel del organigrama. Este intentó fracasó porque las tareas asignadas se tornaron demasiado académicas y complicadas como para poder llevarlas a la práctica. Ahora se prueba desde el extremo opuesto: a cada agencia se le pide con insistencia creciente que informe de los resultados que ha conseguido a partir del presupuesto que tiene asignado. La relación entre departamentos (ministerios), que son los que asignan los fondos, y las agencias ejecutivas se ha organizado en ciclos de tres años. Estas agencias deben emitir cada año un informe simplificado de los resultados obtenidos durante el ejercicio. La descripción se centra aquí casi exclusivamente en los logros. No se pide un análisis exhaustivo de los efectos producidos.

Cada tres años se solicita de las agencias un informe completo de esas repercusiones juntamente con la relación de los resultados que se pretendían conseguir al cabo del mencionado perío do de tres-años.

\section{La asignación de fondos se realiza según un plan financiero trienal}

En el futuro, los fondos destinados a cubrir las necesidades operativas quedarán mucho más claramente ligadas a los informes de gestión y al análisis de resultados. En un principio, el marco financiero se ha diseñado para tres años. Por regla gene$\mathrm{ral}$, las agencias saben de antemano de cuántos recursos dispondrán durante el trienio siguiente, además de contar con la posibilidad de reasignar fondos a lo largo del año económico. Se espera de este modo soslayar dos aspectos negativos: planificar a corto plazo y dedicar demasiada atención al apartado de ingresos en el presupuesto.

\section{Otras modificaciones del sistema financiero}

La gestión por resultados se coordina con diversas reformas del sistema financiero. Un rasgo común en todas estas reformas es que los sistemas financieros gubernamentales se parecen cada vez más a los que utiliza el sector privado. La agencias necesitan créditos para financiar sus inversiones. Se implementan incentivos para una mejor gestión del dinero de caja en las agencias que tienen que pagar intereses por los fondos que no se utilizan en el momento que se había previsto. Los métodos contables se parecen también a los del sector privado porque se asignan costes para áreas operativas y períodos concretos.

\section{La administración de costes dentro de unos limites definidos}

El sistema que se aplicaba hasta ahora se basaba en el supuesto de que las agencias debían ver sus fondos automáticamente incrementados de acuerdo con el aumento de los costes salariales. Con este sistema, no se ha dado ningún incentivo para mantener controlado el nivel salarial. Desde hace un año, todas las agencias financieramente centralizadas han recibido instrucciones relativas a los límites en que se moverán sus costes admi- 
nistrativos. Estos programas marco ya prevén una evolución salarial. Esto quiere decir que, si los salarios de los empleados estatales crecieran por encima de lo previsto, serán los responsables de cada agencia los que tendrán que buscar los fondos adicionales necesarios y variar sus prioridades dentro del marco administrativo impuesto.

Como quiera que a las agencias se les concede cada vez mayor autonomía por lo que hace referencia a las políticas salariales, se estima que los sueldos de los funcionarios crecerán menos de lo que lo venían haciendo. La reforma pretende que los empresarios del sector privado descentralicen el cálculo de salarios y se aplique de forma individualizada en cada empresa, evitando así los convenios colectivos sectoriales.

\section{Políticas de personal adaptadas al mercado}

Los cambios que ha efectuado el gobierno en su política de personal se encuentran entre aquéllos que han dedicado la mayor parte de sus esfuerzos a la implementación. Las líneas maestras se dibujaron hace diez años y, desde entonces, el gobierno está empeñado en romper los valores tradicionales sobre los que han descansado generalmente los sistemas retributivos estatales; valores como el trato uniformado y que el sueldo ha de ser en función del cargo que se ocupa y no de acuerdo a la valía de cada persona. En los sistemas públicos clásicos, la formación de los salarios se decidía en las altas instancias y afectaba a la mayor parte del organigrama administrativo. En Suecia, se han producido dos cambios simultáneos en este sentido:

En primer lugar, la formación de los salarios se decide en instancias inferiores de la Administración nacional, lo que significa que cada agencia tiene la libertad para modelar su régimen de retribuciones. En consecuencia, no existe ya un rasero común en los sueldos que paga la Administración. En segundo lugar, la formación de salarios responde ahora -tal como ocurre en el sector privado- a criterios de valía personal y no de cargo. El sistema de categorías o grados se ha abolido.

Estos cambios han redundado generalmente en políticas salariales más flexibles donde las necesidades del que emplea ejercen un papel predominante. Por un mismo trabajo, se pueden pagar salarios diferentes en distintos lugares, dependiendo siempre del mercado de trabajo local. El patrón tiene la posibilidad de elegir entre varias personas con distintas aptitudes y pagar conforme a las necesidades operativas. También existe, al menos teóricamente, una completa interrelación y movilidad entre los diversos factores de la producción; entre el personal y los demás o, en algunos casos, entre empresarios del sector público y del sector privado.

\section{Resumen de las nuevas condiciones}

El estado de la economía sueca necesita una reducción del gasto público. En primer lugar, esto repercutirá en el sistema de la seguridad social o del bienestar, pero permitirá también, ente otras cosas, tener una organización del Estado más barata mediante, en cierto grado, la corporativización, comercialización, reestructuración y saneamiento de la gestión gubernamental. Las actividades que correspondan finalmente al Estado deberán realizarse de una manera más eficaz. La consecuencia inmediata de este proceso es obvia: el número de empleados estatales disminuirá drásticamente. Tal proceso se inició en los años noventa.

El número de empleados estatales se cifra ahora sobre los 250.000 (se incluye el ejército, la policía, las universidades, etc.). Hace sólo 10 años esta cifra era de 350.000. La corporativización de servicios públicos como las comunicaciones y otras actividades comerciales ha reducido en 72.000 el número de empleados de la Administración. Otros 40.000 han sido despedidos por diversas regulaciones de plantilla y se espera que a esta cifra se le añadan otros 25.000 empleados durante los próximos tres años.

\section{Las organizaciones sindicales cooperan para facilitar los ajustes}

\section{Caso 1: Participación del funcionario en el proceso de trans- formación}

Uno de los efectos de la situación de las finanzas del Estado es la exigencia para obtener una mayor eficacia en la gestión de las organizaciones controladas todavía por la Administración. Esto está muy relacionado con la participación del funcionario en el proceso de cambio. El gobierno ha delegado no pocos poderes a los responsables de las agencias estatales para llevar a cabo sus funciones en la dirección deseada. Esto ha sido posible gracias a la introducción de un nuevo sistema presupuestario basado en la gestión por resultados que permite a los jefes de cada agencia tomar decisiones importantes respecto a la política de personal, organización, fijación de salarios y otras materias que influyen de forma decisiva en el nivel de eficacia de su gestión. Estos directivos tienen libertad para decidir la forma de conseguir el resultado deseado.

\section{Amplia participación de los empleados en la teoria y en la práctica}

Existe en la actualidad todo un sólido conjunto de pruebas empíricas que permite sostener la idea de que el éxito de los cambios viene determinado por la oportunidad que tengan los afectados en participar en el proceso de los mismos. Este hecho tiene relación con la motivación de los empleados para asegurar la permanencia de las reformas y con el uso que se hace de los 
conocimientos de los que serán responsables de llevar a cabo las tareas cotidianas durante tales procesos de transformación.

Éstas son verdades evidentes hoy, pero hace sólo diez o quince años, los intentos por cambiar la Administración central se inspiraban en la opinión exclusiva de un experto y las propias organizaciones de expertos al servicio del Estado formaban un nueva organización por orden del gobierno y establecían los nuevos métodos de trabajo para las agencias que se responsabilizarían del cambio "real". A partir del momento en que el gobierno decidió -bien sea por consejo de los auditores, o por alguna otra razón- que las agencias tenían que ofrecer un mayor nivel de eficacia, no se daba la responsabilidad de la revisión a la agencia afectada, sino que se adjudicaba a expertos en organización de empresas. Hoy, la filosofía dominante manifiesta un deseo por otorgar la mayor parte de la responsabilidad del estudio de reconversión a las mismas agencias; y es también parte de esa filosofía que los empleados colaboren en la misma -una forma de hacer las cosas que presenta dificultades en su aplicación en aquellos casos en que los cambios comportan una reducción del número de empleados.

El Estado y los sindicatos ban creado un fondo de ayuda para las iniciativas de reformas descentralizadoras

Para facilitar el proceso de cambio, el Estado y las organizaciones sindicales del funcionariado crearon en 1987 un fondo destinado a estimular a los organismos estatales para aumentar sus niveles de eficacia. La concepción de este fondo era muy simple. En las negociaciones salariales de conjunto de la Administración, se reservaron 350 millones de coronas suecas para futuros proyectos de transformación y desarrollo. La forma en que se seleccionaron dichos proyectos y tomaron las decisiones sobre los mismos fueron tildadas de únicas, según la opinión internacional. Como los fondos se asignan conforme a acuerdos colectivos, el empresario y el empleado consideran algo natural compartir el poder de decidir el uso que se hará de éstos. El Fondo Nacional para el Desarrollo Administrativo cuenta por consiguiente con tres representantes del Estado y tres representantes de los sindicatos mayoritarios entre los funcionarios. El objetivo consiste en alcanzar un consenso relativo a la asignación de los fondos existentes. Este ha sido el caso en algo más de 700 decisiones tomadas por la Fundación.

Las negociaciones con los empleados resultan esenciales para obtener el apoyo necesario para el proyecto

Otra tarea importante del Fondo en cuanto al valor de la cooperación entre el Estado y los funcionarios es la de decidir qué proyectos gozarán de ayuda financiera y cuáles no. Entre los diversos criterios que se barajan al respecto, está el que los empleados que trabajen en áreas susceptibles de transformación deben poder emitir su opinión en relación a la forma de organización y los objetivos que se pretende conseguir. Para garanti- zarlo, el empresario -es decir, el organismo destinatario del proyecto- debe negociar la organización del proyecto antes de presentar la solicitud al Fondo. Una vez se aprueba el proyecto, deben efectuar otra ronda de consultas antes de iniciar su ejecución.

La participación de los empleados se contempla en el mismo proyecto

Este es sólo el lado formal del asunto. En la mayoría de proyectos, la participación de los empleados se materializa en forma de grupos de trabajo, grupos de organización o de cualquier otra manera parecida. Para un gran número de funcionarios, el haber tomado parte activa en un plan para mejorar su propio puesto de trabajo y los métodos para desempeñarlo ha sido una experiencia nueva. En algunos organismos regionales y locales se ha dado también por primera vez el caso de que los empleados iniciaran un proyecto por propia iniciativa y no siguiendo el tradicional dictado de Estocolmo.

\section{Los cambios organizativos derriban los muros entre profesiones}

La mayor parte de los 400 proyectos tienen como objetivo una ruptura con una idea de organización del trabajo obsoleta que se ha basado a menudo en una estricta división de funciones entre las distintas categorías de empleados. El Estado probablemente sigue el rastro del sector privado porque el sistema salarial del primero ha clasificado a los individuos en función del cargo que ocupan y no de acuerdo con la competencia concreta de cada persona. En muchos lugares se ha reorganizado el trabajo según el grado de conocimiento y experiencia de cada empleado para una tarea determinada. No pocos proyectos han derribado los muros existentes entre el personal directivo y auxiliar (especialmente con los auxiliares).

Cualquier proyecto ahora va más allá de la simple formación de empleados; el Fondo ha rechazado proyectos que consistían pura y exclusivamente en dar más formación al personal. Se pretende con ello un enfoque más abierto y amplio de la cuestión: la revisión de la organización y sus formas de gestión al tiempo que se eleva el nivel de competencia de los que trabajan en ella. Sólo entonces pueden conseguirse cambios permanentes.

Las evaluaciones realizadas indican que los proyectos producen a menudo efectos fuera de la organización en que se llevan a cabo. Son también una fuente de inspiración para otros lugares de trabajo. Como ejemplo de ello, cabe mencionar las pruebas que se realizan en diversos departamentos de la universidad con criterios organizativos diferentes. Se empieza a notar ahora el interés de otras universidades que no participaron anteriormente en el desarrollo del proyecto.

\section{Dificultades para difundir las experiencias}

Incluso en el caso de que uno observe que una experiencia con éxito se extiende a otras instancias, debe esperarse 8 años 
para su reconocimiento definitivo porque entretanto pueden aparecer otras ideas válidas en la misma dirección. El Fondo ha modificado progresivamente sus métodos de trabajo en función de esta circunstancia. El método original consistía en que las agencias solicitaran las ayudas correspondientes para el desarrollo de sus proyectos casi de la misma forma que lo hacía un instituto de investigación o se pedían fondos para un determinado presupuesto.

Los nuevos proyectos se crean para que las agencias intercambien sus experiencias

El Fondo desea ahora que la experiencia se comparta desde el inicio mismo de cada proyecto. Por esta razón, se intenta evitar la concesión de ayudas para proyectos individuales destinados a agencias concretas. La filosofía de las ayudas es financiar programas que contemplen la reforma de algún asunto de interés en el que puedan cooperar varias agencias y así verificar de una forma más efectiva los métodos de trabajo y revertir los beneficios conseguidos a la sociedad en general.

El ejemplo: menos tiempo para despachar los asuntos presentados a la consideración de las agencias

El primer test de este método se realizó en una provincia hace seis años. Era público y notorio que la gente en general estaba descontenta de lo poco que hacían las agencias para acortar el tiempo que tardaban en despachar los asuntos que se les presentaban. Paralelamente, se sabía que el 90 por ciento del tiempo que tardaba un asunto en ser tratado se consumía de hecho en las esperas que se producian mientras el caso pasaba de una mano a otra. La cuestión era cómo reorganizar ese 90 por ciento del tiempo en que no ocurría nada más que saber qué pasaba cuando alguien trabajaba en ello.

La Oficina Nacional de Auditorías ya había elaborado diversos métodos para este caso, pero nunca se habían aplicado. El Fondo ofreció entonces a algo así como organismos regionales y locales (desde comisarías de policía a agencias de colocación) la oportunidad de tomar parte en una especie de concurso para ser los mejores del país en cuanto a acelerar los trámites de los asuntos con que trataban. La mayoría aceptó el reto. En la mayor parte de los casos se pudo observar que las agencias redujeron los tiempos de espera a la mitad al cabo de un año. Algunos de estos casos sirvieron de ejemplo para las organizaciones hermanas de otras provincias.

Los programas "multi-agencia tocan aspectos críticos del desarrollo administrativo

El mismo enfoque ha servido para un buen número de casos semejantes. El Fondo ha participado en proyectos sobre la organización del trabajo, el desarrollo operativo y la igualdad entre sexos -también con resultados positivos. En la actualidad se está elaborando un programa de gran alcance sobre mejora de la gestión dirigido exclusivamente a mujeres con responsabilidades directivas.

En todos los casos, el personal afectado se implicó muy activamente en el proyecto. Los asesores actuaron solamente como referencia y no dirigieron su evolución. Los proyectos fueron dirigidos por personas que mostraron un especial interés por el tema que se planteaba y se eligieron de entre el personal de cada rama en las agencias implicadas.

La necesidad de mejorar los conocimientos sobre la gestión del cambio es ahora mayor porque los procesos de transformación parten ahora de las mismas agencias. Consecuentemente, el Fondo ha iniciado una serie de programas para mejorar la competencia de las agencias en el diseño y puesta en marcha de los procesos de cambio. Parte de esta estrategia ha sido el establecimiento de una Academia del Cambio dirigida al personal de mayor antigüedad con responsabilidad directa en el proceso de transformación de su propia agencia. En el programa participan los mejores expertos del país.

\section{El Fondo y el futuro de un trabajo en común}

El Fondo concentrará sus esfuerzos en un cierto número de áreas consideradas de vital importancia para el futuro de la Administración central en Suecia. Entre éstas se cuenta preparar a la Administración para los efectos que producirá la internacionalización, un tema que ha ganado en trascendencia después del ingreso de Suecia en la Unión Europea. Muchos consideran que la "internacionalización", con todas sus facetas, nos obligará a efectuar transformaciones mucho más drásticas que las producidas en estos últimos diez años. Es de prever la creación de una división del trabajo entre agencias europeas hermanas, unas veces para competir entre sí y otras para trabajar de forma cooperativa. En este campo, es probable que las instituciones suecas dedicadas a la transformación administrativa se beneficien de esta ampliación de las fronteras para incorporar también otras agencias extranjeras además de las nacionales.

El Fondo tiene interés también en garantizar que el proceso de gestión por resultados que se ha implementado ahora no se limite únicamente en cuestiones técnicas que afectan sólo a los departamentos financieros, sino que tome el cuerpo adecuado para llegar a todo el personal de la agencia. De esta manera, todo lo que se ha logrado ya puede servir como semilla para que los cambios se generen a nivel local. Si alguien puede tener una visión y una medida de lo que logra con los recursos disponibles, es posible evaluar si su trabajo puede hacerse mejor.

Caso 2: Los sindicatos y el Estado acuerdan las condiciones de despido cuando se prescinde de personal

El modelo tradicional de empleo público significaba que el titular de un puestø de trabajo, al menos para los empleados de más antigüedad, podía contar con un considerable grado de segu- 
ridad. Tal idea ya se ha abandonado hoy en día. En el presente, el empleo público no resulta muy distinto al que puede obtenerse en la industria o en cualquier otra área del mercado laboral. Los cambios se han sucedido con rapidez y los sindicatos que agrupan a los funcionarios han aceptado las nuevas condiciones. No obstante, esta aceptación ha exigido un precio: el Estado ha acordado con los sindicatos pactar las condiciones de despido.

Esta solución resulta probablemente singular. El Estado y los sindicatos han acordado las condiciones que deben aplicarse en caso de despido y deciden crear solidariamente una Fundación con el sólo propósito de trabajar para encontrar un nuevo empleo, en la medida de lo posible, a los trabajadores despedidos.

\section{Las bases del sistema regulador}

El sistema regulador no hace diferencias entre el caso en que el funcionario recibe una nueva colocación en la Administración pública y el caso en que lo obtiene en cualquier otro sector del mercado de trabajo. Lo que importa es que el trabajador consiga un trabajo adecuado.

Como resultado de este acuerdo, para la mayoría de funcionarios media un período de seis meses a un año entre la notificación del despido y efectividad del mismo. Durante este lapso de tiempo se le ofrecen generosamente diversas oportunidades, liberándole de sus obligaciones, para que adquiera nuevos conocimientos y busque otro empleo.

A fin de eliminar los obstáculos que pudieran derivarse de las distintas condiciones laborales, el acuerdo contiene dos cláusulas destinadas a facilitar la movilidad de empleo:

La primera hace mención a la seguridad de los ingresos: aquéllos que acepten un nuevo puesto de trabajo tienen derecho a una compensación durante los primeros cuatro años en el nuevo puesto si el salario de este último fuera inferior al que abandonan. Esta compensación se cifra como máximo hasta un 30 por ciento del salario anterior.

La segunda se refiere a la llamada uprotección ante el después", que quiere decir que si un funcionario acepta un nuevo puesto y lo pierde luego, recibirá el mismo trato que un empleado que acaba de perder su empleo en la Administración y, por tanto, será beneficiario de todos los acuerdos sobre seguridad en el empleo. Esta protección tiene una vigencia de cinco años, siempre y cuando que el nuevo empleo no disfrute de un convenio equivalente en este campo al que tenía en la Administración.

Una Fundación creada por las partes para obtener nuevo empleo a los despedidos

La parte más interesante del acuerdo se centra posiblemente en el trabajo de la Fundación, que cuenta con dos tipos de recursos: en primer lugar, una considerable suma de capital para financiar sus gestiones. Este fondo se nutre cada año de un por- centaje sobre el volumen salarial global desembolsado. En segundo lugar, un equipo muy capaz de asesores que tratan con cada afectado de forma individualizada.

A diferencia de lo que es usual en las políticas de personal de la Administración, el trabajo de la Fundación no está regulado, si no es en términos muy generales. Dentro de unos límites razonables, la Fundación está para hacer todo lo posible para conseguir un nuevo empleo a cada afectado por un despido. Este es su objetivo y su función. El equipo asesor conjuntamente con el empleado, su nuevo empresario y las oficinas de empleo puede usar libremente los fondos de que dispone la Fundación. A diferencia también de los convenios colectivos tradicionales, el empleado no puede arrogarse ante la Fundación ningún derecho en concreto. No hay normas fijas, manda la situación práctica.

Las medida más común es ofrecer cursos de formación en aquellas áreas que se consideren favorables para obtener un nuevo empleo, como por ejemplo los cursos para iniciar un negocio por cuenta propia. En este sentido, la Fundación facilita la participación de expertos del sector pertinente para que asesoren a los nuevos empresarios.

La Fundación puede llegar a pagar un salario a un empleado durante el período de prueba en una nueva ocupación. Durante ese tiempo decidirá si el trabajo es adecuado para él e intentará demostrar a los empresarios escépticos que la experiencia de un funcionario también puede resultar de utilidad.

\section{Compensación para aquéllos que no consiguen empleo}

La misión más importante de la Fundación es ayudar a los despedidos por el Estado a encontrar un nuevo empleo. Existe asimismo un sistema de compensaciones que cubre el supuesto en que la gente no encuentre ocupación. Si el trabajador tiene 60 años o más, tendrá derecho a recibir una pensión igual a la que hubiera recibido de retirarse a los 65 .

En los otros casos, se mejoran las prestaciones generales del seguro de desempleo hasta alcanzar el 80 por ciento del salario anterior al despido. En circunstancias muy particulares, esta prestación especial puede extenderse hasta un período de cinco años, si bien resulta infrecuente. En la mayoría de ocasiones, la persona afectada resuelve ella misma la situación y encuentra trabajo mucho antes.

\section{Comentarios finales}

A pesar de que el número de empleados públicos se ha reducido en una proporción impensable hace tres o cuatro años, todavía hay fe en Suecia en el valor de la colaboración entre empresarios y sindicatos dentro de la Administración. Los funcionarios y sus organizaciones representativas se han dado cuenta de que es necesario ofrecer una mayor eficacia en los servicios. Consideran que es mejor poder participar e influir en los 
cambios que oponerse y dejar a los empresarios solos con la responsabilidad y el poder de decisión.

El proceso de reducción de personal se ha tratado de una forma muy razonable. La garantía de ello es que todas las partes controlan de forma paritaria los órganos que facilitan los servicios de recolocación.
A pesar de que muchos aspectos del modelo sueco se están volviendo a examinar en la actualidad, permanece la convicción de que cooperar para mejorar la organización y la Administración resulta beneficioso. Este es uno de los valores que seguirán vigentes en Suecia después del período de ajustes más doloroso desde los años treinta.

Artículo traducido por Josep M. CARRERAS I SANAHUJA.

* Director del Fondo Nacional para el Desarrollo Administrativo, Ministerio de Finanzas, Suecia. 


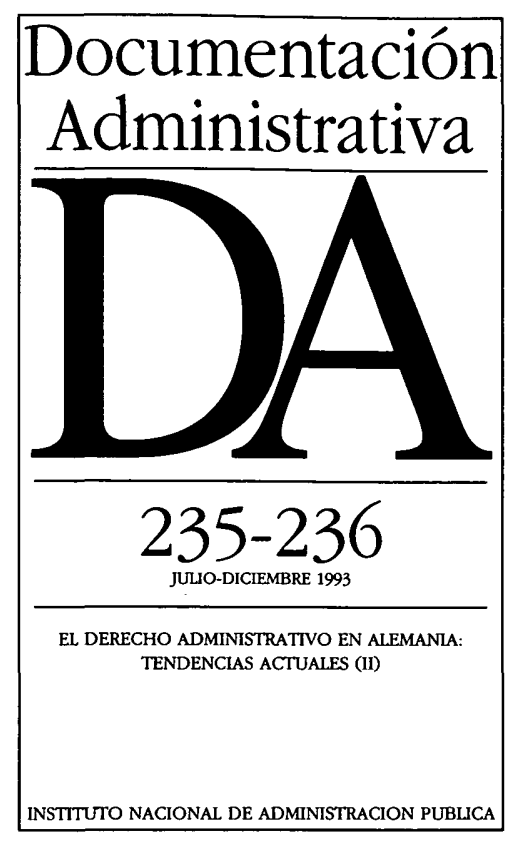

Publicación trimestral del Instituto Nacional de Administración Pública, especializada en Derecho Administrativo y Organización y Gestión de las Administraciones Públicas.

Aparecida en 1958, la característica más destacada de su actual etapa es la dedicación monográfica de cada número a una cuestión específica, que se analiza desde diferentes puntos de vista por acreditadas firmas de España y otros países.

\section{CONSEJO DE REDACCION}

Presidente: Alejandro Nieto García. Vocales: Manuel Aragón Reyes, Enrique Argullol Murgadas, Miguel Beltrán Villalva, Andrés de Blas Guerrero, José Manuel Castells Arteche, Rafael Gómez-Ferrer Morant, Luis Morell Ocaña, José Ramón Parada Vázquez, Juan Alfonso Santamaría Pastor, Gumersindo Trujillo Fernández.

\section{Director}

Luciano Parejo Alfonso

Secretario

Jesús Prieto de Pedro

\section{Edita:}

Instituto Nacional de Administración Pública

ISSN: 0012-4494

NIPO: 329-94-001-5

Depósito legal: M 581-1958

\section{Distribución y suscripciones:}

Instituto Nacional de Administración Pública Subdirección General de Documentación, Investigación y Publicaciones José Marañón, 12. 28010 MADRID Teléfono: (91) 4461700 Fax: (91) 4450839

\section{Precios:}

Suscripción anual (4 números) 4.000 pts. + IVA

Número sencillo. 1.300 pts. + IVA

Número doble

2.600 pts. + IVA

Más 300 pts. de gastos de envío 